\title{
Clinicopathological Features And Prognosis of Primary Pulmonary Rhabdomyosarcoma In The Middle-Aged And Elderly: A Case Report And Literature Review
}

\author{
Wei Yingze \\ Department of Pathology, Nantong Tumor Hospital \\ Jin Xiaoxia \\ Department of Pathology, Nantong Tumor Hospital \\ Wang Jiatai \\ Department of Pathology, Nantong Tumor Hospital \\ Zhu Xinghua \\ Department of Pathology, Nantong Tumor Hospital \\ Chen Xudong ( $\nabla$ ntchenxudong@163.com ) \\ Department of Pathology, Nantong Tumor Hospital \\ Yang Shuyun \\ Department of Pathology, Nantong Tumor Hospital
}

\section{Case report}

Keywords: primary, pulmonary, rhabdomyosarcoma, clinicopathological, prognosis, middle-aged and elderly

Posted Date: November 23rd, 2021

DOI: https://doi.org/10.21203/rs.3.rs-948442/v2

License: (c) (i) This work is licensed under a Creative Commons Attribution 4.0 International License. Read Full License 


\section{Abstract}

Background: Primary pulmonary rhabdomyosarcoma囚PPRMS囚in the middle-aged and elderly is a very rare event with only a few cases published. To date, only thirty-five cases of PPRMS in the middle-aged and elderly have been published. However, the analyses on clinicopathological characteristics and prognosis of PPRMS in the middle-aged and elderly have not been performed.

Case presentation: We report an additional case of PPRMS in the middle-aged and elderly.

Conclusions: PPRMS in the middle-aged and elderly was a highly malignant soft tissue tumor with obvious gender characteristics and significant age distribution. Pleomorphic rhabdomyosarcoma (RMS) was the most common subtype, with poor prognosis and significantly prolonged survival time after surgical resection.

\section{Background}

RMS is a malignant primitive mesenchymal tumor with skeletal muscle differentiation and it is the most common sarcoma in children, accounting for more than $50 \%$ of soft tissue sarcomas in those age groups, but less than $5 \%$ of sarcomas in adults [1-3]. RMS usually occurs in the head and neck (35\%), the urogenital system (21\%), the extremities (19\%), and the chest (7\%) [4]. The clinicopathological features and prognosis of 76 RMS cases in the middle-aged and elderly $\geq 40$ years old were analyzed, of which only 2 cases occurred in the lung [5], hence on PPRMS in the middle-aged and elderly is rarer. At present, the studies on clinicopathological features and prognosis of PPRMS in the middle-aged and elderly have not been reported.

In this study, we first reported a case of pulmonary acinar RMS in the middle-aged and elderly $\geq 45$ years old which has been corrected to define as middle-aged and elderly according to the latest definition of the World Health Organization (WHO), and then retrospectively reviewed 35 cases of PPRMS in the middleaged and elderly reported in PubMed and Elsevier websites, finally analyzed the clinicopathological characteristics and prognosis of 36 cases of PPRMS including this present reporting case, thus in order to have a general understanding of PPRMS in the middle-aged and elderly for clinicians and pathologists.

\section{Case Presentation}

Clinical findings

A 75-year-old man came to our hospital because of abdominal pain and discomfort. There were no obvious abnormalities in blood routine and biochemical indexes, except the increase of lactate dehydrogenase (LDH), which was 442 (normal: 106-211 U / L). The serum tumor markers carcinoembryonic antigen (CEA), cytokeratin 19 fragment (CYFRA21-1) and squamous cell carcinoma antigen (SCC) were normal, while the neuron specific enolase (NSE) and progastrin releasing peptide (proGRP) were increased, and the values were 63.07 (normal: 0-17) ng / $\mathrm{ml}$ and 84.68 (normal: 25.3-69.2) pg 
/ $\mathrm{ml}$, respectively. Positron emission tomography-computer tomography (PET-CT) showed a lobulated mass of $7.6 \mathrm{~cm} * 5.5 \mathrm{~cm}$ in the lower lobe of the left lung, with abnormally high fluoro-2-deoxy-D-glucose (FDG) metabolism (Fig. 1)

\section{Pathological findings}

Histologically, the left lung biopsy showed the tumor cells were small, with few cytoplasm, deep staining of nuclei and heavy stained nuclear chromatin, very similar to the morphology of small cell lung carcinoma (SCLC) (Fig. 2a). Immunohistochemically, the tumor cells stained positive for desmin, MyoD1 and myogenin, synaptophysin (Syn), CD56 (Fig. 2b-f), SMARCA4 and Ki67 index was 90\% positive. Negative staining was for epithelial markers CK (AE1 / AE3), CK18, EP cam, CK (cam5.2), CK7, CK8 and CK14, and other markers such as TTF-1, chromogranin A (Cg A), FLI-1, CD99, CD20, CD3 and Pax5, HMB45, S-100, NUT, SALL4, SOX2, CD34. Cytogenetic analysis for FOX01A translocation was negative. The diagnosis of acinar PPRMS was confirmed by Prof. Wang Jian from the pathology department of fudan university shanghai cancer center.

Therapy and outcome

RMS is usually treated by surgery, chemotherapy and / or radiotherapy[5]. Because of his general weakness condition, the patient could not bear tumor resection, and was given a combined chemotherapy of VAC regimen [Vincristine $1 \mathrm{mg}+$ actinomycin $0.4 \mathrm{mg}+$ cyclophosphamide $0.8 \mathrm{mg}$ ], however, the patient only received one course of chemotherapy and died 2 months after the diagnosis.

\section{Discussion And Conclusions}

Due to the rarity of PPRMS in the middle-aged and elderly, information regarding its clinicopathological characteristics and prognosis is very limited. The large, multi-institutional trials have not been performed, and only reports from single institutions have been published. More importantly, so far the analyses on clinicopathological characteristics and prognosis of PPRMS in the middle-aged and elderly have not been performed.

RMS occurred in the lung, there was no specificity in clinical manifestation and serum laboratory examination. For our case, we found LDH, NSE and Pro-GRP in his blood were simultaneously elevated, these three markers useful in diagnosis and predicting the prognosis of SCLC[6-8]. Under microscopy, the tumor cells were small, deeply stained with extrusion deformation, which should be distinguished from some small round cell tumors occurred in lung: (1) Both serological data, microscopic morphology and immunohistochemical results of neuroendocrine markers were easily misdiagnosed as SCLC, because it was more common than RMS in lung. Moreover, for RMS, immunohistochemical results also partially overlapped with SCLC, since $43 \%$ of acinar PPRMS expressed at least one specific neuroendocrine marker, 32\% expressed Syn, 22\% expressed CgA, 11\% expressed both of them, and CD56 was expressed in almost all cases[9]. However, the epithelial markers usually were positive and TTF-1 was positively expressed up to $80-90 \%$ in SCLC[10]. (2) Morphologically, monomorphous small or medium size cells with 
scant cytoplasm may not be completely distinguished from NUT carcinoma which is an aggressive malignancy and often occurs at the mediastinum and the upper aerodigestive tract in patients of any age with variable symptoms at presentation depending on anatomic location. However, NUT carcinoma often exists foci of abrupt squamous differentiation with keratinization scattered among the more primitivelooking cell nests. Immunohistochemically, the tumor cells are commonly positive for NUT, CK5/6, P63, P40 and other markers including CD99, Fli-1, Syn, EGFR, and HER2[11]. Our immunohistochemical results ruled out the diagnosis of NUT carcinoma. (3) Thoracic SMARCA4-deficient undifferentiated tumors are also aggressive malignancies diagnosed more often in young male smokers with undifferentiated morphology with occasional hepatoid and rhabdoid features. Immunohistochemically, the tumor cells are often negative for SMARCA4, concurrent loss expression of SMARCA2, while positive for one or more stem cell markers SOX2, CD34, or SALL4 [11]. Our immunohistochemical results also ruled out the diagnosis of this tumor. (4) Pleuropulmonary blastoma (PPB) may also not be distinguished from RMS, but the former is a rare and typically occurring in children less than 6 years of age. PPB is divided into subtypes I, Ir, II, and III. Type I is a purely cystic lesion, and type II tumors have cystic and solid components, and type III are purely solid lesions. It is associated with the germline mutation in DICER1. On HE, PPB showed two components, comprised of small round uniform hyperchromatic blue cells and the other comprised of occasional large cells having central nucleus and moderate cytoplasm. On IHC, the hyperchromatic blue cells were positive for vimentin and occasional large cells showed immunoreactivity for Pan CK. Our immunohistochemical results also ruled out the diagnosis of this tumor $[12,13]$. (5) Some other rare tumors such as soft tissue clear cell sarcoma, extraosseous Ewing sarcoma, non-Hodgkin lymphoma, and malignant melanoma, etc, resembled acinar RMS, but immunohistochemical markers of those tumors such as HMB45, S-100, CD99, Fli-1, CD20, CD3 and Pax5 were all negative. (6) PET-CT showed no other lesions in his whole body, ruling out secondary tumors.

To better characterize the clinical features and outcomes of PPRMS in the middle-aged and elderly, we searched all PPRMS in the middle-aged and elderly from 1958 to 2021 on PubMed and Elsevier websites and removed the repeated cases in these literatures. We performed an analysis of 36 PPRMS cases which consisted of 35 cases collected from PubMed and Elsevier websites[14-22] and one case in our own hospital (Table. 1). In a total of 36 cases, males were significantly more than females $(P=0.001)$. The incidence of 50-69 years old was higher than that of $45-49$ years old and $\geq 70$ years old $(P=0.000)$; Pleomorphic type was more common than embryonal and acinar type $(P=0.000)$ (Table. 2). 35 patients received different treatment methods. Among the 34 patients followed up, there was a significant correlation with the prognosis. The survival time of supportive treatment was short and the survival time of surgical resection was significantly prolonged $(P=0.010)($ Fig.3).

We found that there was a significant gender difference and notable age distribution in PPRMS with the middle-aged and elderly, most of them were men and often occurred between the ages of 50-69 years old. Due to the rarity and only some published reports of PPRMS in the middle-aged and elderly, there has been no similar opinion at present. A previous study found the ratio of men to women is 1.5:1 in 76 RMS patients $\geq 40$ years old[5]. A recent study also showed 66 patients with embryonal and alveolar RMS in adults included 42 men and 24 women[23]. A large study analyzed data from RMS with 1,071 adults (age 
$>19$ years) and 1,529 children (age $\leq 19$ years) and males were more than females too among both of these adults and children[3]. Some researchers found that male predominance (male/female of 1.5:1) was noticed for almost all types of soft tumor sarcomas and the age range $20-76,25-71,18-86$, and 55-76 years old for head and neck primaries, genitourinary primaries, limbs, or other primary sites, respectively $[24,25]$.

There was a significant difference in histological type of PPRMS with the middle-aged and elderly, most of them were diagnosed as pleomorphic type. A large study pointed out that embryonal and alveolar subtype were the most and second common in children/adolescents, while embryonal and pleomorphic subtype other than not otherwise specified (NOS) subtype were the two most common in adults[3]. A previous study on 76 cases of RMS in the middle-aged and elderly also proposed that embryonal and pleomorphic subtype were the two most common[5]. Another present study found for pleomorphic RMS, the incidence was more frequently and the median age of 51 years was older than alveolar and embryonal RMS[25]. Pleomorphic RMS is an aggressive sarcoma, arising predominantly in the extremities of adult males usually 45 of older[26]. Similar with the above studies, PPRMS in the middleaged and elderly were more males as well as pleomorphic predominant subtype.

The combination of surgery, chemotherapy and / or radiotherapy has achieved good results in children's RMS, and the prognosis of children's rhabdomyosarcoma has been significantly improved. The successful experience in the treatment of RMS in children has been widely used in the treatment of adult RMS (including middle-aged and elderly), but the effect is not good[5]. Polymorphic RMS is less sensitive to chemotherapy, and its treatment depends more on surgery. The unfavorable prognostic factors of RMS are anatomical location such as parameningeal, extremities, abdomen, chest and so on, histological subtypes including acinar and pleomorphic RMS, tumor size $>5 \mathrm{~cm}$, as well as metastasis[27]. In this study, although some of patients received surgery, and or chemotherapy, 26 of 36 cases were diagnosed as pleomorphic type and acinar type, and each case was located in lung, the prognosis of PPRMS in the middle-aged and elderly was poor.

Our analyses were not without limitations. First, as most of cases from literatures spanned a long period of time, progress in supplementary examination shifted the diagnosis of RMS from standard morphological detection to incorporate immunohistochemical staining, and now to molecular testing. It is possible that some of cases included in our analyses were not RMS, particularly those with pleomorphic type. Second, not every case had details of clinicopahtological features and prognosis such as tumor size, as a result, some clinicopathological features such as tumor size could not be statistically analyzed.

In a word, we reported a case of acinar PPRMS in the middle-aged and elderly, and retrospectively reviewed and analyzed the clinicopathological features of PPRMS in these middle-aged and elderly, so as to help clinicians and pathologists better understand this rare tumor of the lung in the middle-aged and elderly.

\section{Abbreviations}


PPRMS, primary pulmonary rhabdomyosarcoma; RMS, rhabdomyosarcoma; WHO world health organization; LDH, lactate dehydrogenase; CEA, carcinoembryonic antigen; CYFRA21-1, cytokeratin 19 fragment; SCC, squamous cell carcinoma antigen; NSE, neuron specific enolase; pro-GRP, progastrin releasing peptide; PET-CT, positron emission tomography-computer tomography; FDG, fluoro-2-deoxy-Dglucose; SCLC, small cell lung carcinoma; Syn, synaptophysin; Cg A, chromogranin A.

\section{Declarations}

Acknowledgements

Declared none.

Authors' contributions

The patient of this case report was treated by Yang shuyun. Histopathology was performed by Chen xudong and Yang shuyun. Wang jiatai and Zhu Xinghua was contacted for immunohistochemical and molecular pathological analysis. The manuscript was drafted by Wei yingze and Jin xiaoxia with contribution of all coauthors. The final manuscript was read and agreed upon by all authors.

\section{Funding}

There was no funding for this article.

Ethics approval and consent to participate

This study was performed in line with regulations issued by the National Health Commission of China and was approved by the local Commission of Ethics (reference number 2021-A 10). Informed consent was obtained from the participant included in the study.

Consent for publication

Written informed consent was obtained from the patient for publication of this case report and any accompanying images.

Competing interests

The authors declare that they have no competing interests.

Availability of data and material

Data and materials of this work are available on request by the corresponding authors.

Author details

Department of Pathology, Nantong Tumor Hospital, Nantong, Jiangsu, China 


\section{References}

1. Rudzinski ER, Kelsey A, Vokuhl C, Linardic CM, Shipley J, Hettmer S, et al. Pathology of childhood rhabdomyosarcoma: A consensus opinion document from the Children's Oncology Group, European Paediatric Soft Tissue Sarcoma Study Group, and the Cooperative Weichteilsarkom Studiengruppe. Pediatr Blood Cancer. 2021; 68(3):e28798.

2. Leiner J, Le Loarer F. The current landscape of rhabdomyosarcomas: an update. Virchows Arch. 2020;476(1):97-108.

3. Sultan I, Qaddoumi I, Yaser S, Rodriguez-Galindo C, Ferrari A. Comparing adult and pediatric rhabdomyosarcoma in the surveillance, epidemiology and end results program, 1973 to 2005: an analysis of 2,600 patients. J Clin Oncol. 2009;27(20):3391-3397.

4. Chekhlabi N, Toughza J, Dini N. Primary Rhabdomyosarcoma of the Pleura: A Case Report and Review of the Literature. Cureus. 2021; 13(1):e12491.

5. Yu L, Wang J. Rhabdomyosarcoma in middle to old-aged patients: analysis of clinicopathological features and prognosis in 76 cases. Zhonghua Zhong Liu Za Zhi. 2012;34(12):910-916.

6. Barchiesi V, Simeon V, Sandomenico C, Cantile M, Cerasuolo D, Chiodini P, et al. Circulating progastrinreleasing peptide in the diagnosis of Small Cell Lung Cancer (SCLC) and in therapeutic monitoring. J Circ Biomark 2021;10:9-13.

7. Wang $\mathrm{C}$, Jin S, Xu S, Cao S. The combination of pretreatment prognostic nutritional index and neuronspecific enolase enhances prognosis predicting value of small cell lung cancer. Clin Respir J. 2021;15(3):264-271.

8. Yu J, Du F, Yang L, Chen L, He Y, Geng R, et al. Identification of potential serum biomarkers for simultaneously classifying lung adenocarcinoma, squamous cell carcinoma and small cell carcinoma. Cancer Biomark. 2021;30(3):331-342.

9. Bahrami A, Gown AM, Baird GS, Hicks MJ, Folpe AL. Aberrant expression of epithelial and neuroendocrine markers in alveolar rhabdomyosarcoma: a potentially serious diagnostic pitfall. Mod Pathol. 2008;21(7):795-806.

10. Hokari S, Tamura Y, Kaneda A, Katsura A, Morikawa M, Murai F, et al. Comparative analysis of TTF-1 binding DNA regions in small-cell lung cancer and non-small-cell lung cancer. Mol Oncol. 2020;14(2):277293.

11. Chatzopoulos K, Boland JM. Update on genetically defined lung neoplasms: NUT carcinoma and thoracic SMARCA4-deficient undifferentiated tumors. Virchows Arch. 2021; 478(1):21-30. 
12. Bhalerao S, Adhav A, Gandhe S, Nagarkar R. Metachronous pleuropulmonary blastoma in an adult patient with endometrial cancer: a case report. Oxf Med Case Reports. 2019; 2019(6):omz056.

13. Bownes LV, Hutchins SC, Cardenas AM, Kelly DR, Beierle EA. Pleuropulmonary blastoma in an adolescent. J Pediatr Surg Case Rep. 2020; 59.

14. Comin CE, Santucci M, Novelli L, Dini S. Primary pulmonary rhabdomyosarcoma: report of a case in an adult and review of the literature. Ultrastruct Pathol. 2001;25(3):269-273.

15. Kida Y, Katakami N, Tomii K, Ishihara K, Takahashi Y, Imai Y. Case of rapidly progressing primary pulmonary rhabdomyosarcoma with bloody pleural effusion. Nihon Kokyuki Gakkai Zasshi. 2009; 47(5):404-409.

16. Klepetko W, Wisser W, Birsan T, Mares P, Taghavi S, Kupilik N, et al. T4 lung tumors with infiltration of the thoracic aorta: is an operation reasonable? Ann Thorac Surg. 1999;67(2):340-344.

17. Grouls V, Helpap B. Pulmonary rhabdomyosarcoma (author's transl). Thoraxchir Vask Chir. 1976; 24(2):94-97.

18. Ji GY, Mao H. Primary pulmonary rhabdomyosarcoma in an adult: a case report and review of the literature. J Zhejiang Univ Sci B. 2013; 14(9):859-865.

19. Qu Geping, Xiu Qingyu, Li Bing, Shi Zhaoquan. Primary pulmonary rhabdomyosarcoma in adult: A case report. Journal of Medical Colleges of PLA. 2009; 24(06):370-372.

20. Nishioka Y, Tane S, Nishio W, Tanaka H, Ogawa H, Kitamura Y, et al. A rare resected case of pulmonary rhabdomyosarcoma. Gen Thorac Cardiovasc Surg. 2019; 67(12):1089-1092.

21. Chaddha U, Hagen JA, Yaghmour B. Pulmonary Rhabdomyosarcoma Associated with Check-Valve Mechanism. Am J Respir Crit Care Med. 2017; 196(3):e7-e9.

22. Mehmood QU, Shaktawat SS, Parikh O. Case of rhabdomyosarcoma presenting with myasthenia gravis. J Clin Oncol. 2011; 29(22):e653-655.

23. Drabbe C, Benson C, Younger E, Zaidi S, Jones RL, Judson I, et al. Embryonal and Alveolar Rhabdomyosarcoma in Adults: Real-Life Data From a Tertiary Sarcoma Centre. Clin Oncol (R Coll Radiol). 2020; 32(1):e27-e35.

24. Ferrari A, Sultan I, Huang TT, Rodriguez-Galindo C, Shehadeh A, Meazza C, et al. Soft tissue sarcoma across the age spectrum: a population-based study from the Surveillance Epidemiology and End Results database. Pediatr Blood Cancer. 2011; 57(6):943-949.

25. Bompas E, Campion L, Italiano A, Le Cesne A, Chevreau C, Isambert N, et al. Outcome of 449 adult patients with rhabdomyosarcoma: an observational ambispective nationwide study. Cancer Med. 2018; 
7(8):4023-4035.

26. Ruiz-Mesa C, Goldberg JM, Coronado Munoz AJ, Dumont SN, Trent JC. Rhabdomyosarcoma in adults: new perspectives on therapy. Curr Treat Options Oncol. 2015; 16(6):27.

27. Kerin U, Wolohan C, Cooke K. Rhabdomyosarcoma: an overview and nursing considerations. $\mathrm{Br} \mathrm{J}$ Nurs. 2018; 27(6):328-332.

\section{Tables}

Table 1 PPRMS in the middle-aged and elderly (Review of the literature) 


\begin{tabular}{|c|c|c|c|c|c|c|c|}
\hline Patients & Sex & $\begin{array}{l}\text { Age } \\
\text { ( } \\
\text { years) }\end{array}$ & Location & Histology & Follow-up & Therapy & Ref. \\
\hline \multirow[t]{12}{*}{12} & $M$ & 52 & $\begin{array}{l}\text { Right } \\
\text { lobe }\end{array}$ & Pleomorphic & DOD(5months) & SUP & 11 \\
\hline & $M$ & 68 & Left lobe & Pleomorphic & DOD(1months) & SUP & \\
\hline & $M$ & 56 & Left lobe & Pleomorphic & DOD(5months) & $S$ & \\
\hline & $M$ & 53 & Left lobe & Pleomorphic & DUD (152months) & $S$ & \\
\hline & $M$ & 55 & Left lobe & Pleomorphic & AWD (40months) & S & \\
\hline & $\mathrm{F}$ & 66 & $\begin{array}{l}\text { Right } \\
\text { lobe }\end{array}$ & Pleomorphic & NED (40months) & $S$ & \\
\hline & $\mathrm{M}$ & 68 & Left lobe & Pleomorphic & NED (36months) & S & \\
\hline & $M$ & 69 & Left lobe & Pleomorphic & $\mathrm{DOD}(0.67$ months $)$ & SUP & \\
\hline & $M$ & 78 & Left lobe & Pleomorphic & DOD (<1month) & SUP & \\
\hline & M & 58 & Left lobe & Pleomorphic & DOD (<1month) & SUP & \\
\hline & $M$ & 57 & Left lobe & Pleomorphic & DOD(24months) & SUP & \\
\hline & $M$ & 62 & Left lobe & Pleomorphic & NED (24months) & $\mathrm{S}+\mathrm{RT}$ & \\
\hline \multirow[t]{11}{*}{16} & $M$ & 64 & $\begin{array}{l}\text { Right } \\
\text { lobe }\end{array}$ & Pleomorphic & DOD(1months) & SUP & 12 \\
\hline & M & 61 & $\begin{array}{l}\text { Right } \\
\text { lobe }\end{array}$ & NS & DOD(1months) & SUP & \\
\hline & $M$ & 65 & Left lobe & NS & DOD(2months) & SUP & \\
\hline & $\mathrm{F}$ & 71 & $\begin{array}{l}\text { Right } \\
\text { lobe }\end{array}$ & Pleomorphic & DOD(2months) & $S$ & \\
\hline & $\mathrm{F}$ & 55 & $\begin{array}{l}\text { Right } \\
\text { lobe }\end{array}$ & Pleomorphic & AWD(12 months) & S & \\
\hline & $M$ & 65 & Left lobe & Pleomorphic & DOD(3months) & SUP & \\
\hline & M & 64 & $\begin{array}{l}\text { Right } \\
\text { lobe }\end{array}$ & Pleomorphic & DOD(2months) & SUP & \\
\hline & $F$ & 61 & Left lobe & NS & DOD(2 months) & SUP & \\
\hline & $F$ & 48 & Left lobe & NS & AWD(18 months) & NS & \\
\hline & $\mathrm{M}$ & 79 & $\begin{array}{l}\text { Right } \\
\text { lobe }\end{array}$ & Pleomorphic & NS & NS & \\
\hline & $\mathrm{M}$ & 64 & Left lobe & embryonal & DOD(2 months) & $S$ & \\
\hline
\end{tabular}




\begin{tabular}{|c|c|c|c|c|c|c|c|}
\hline & M & 68 & $\begin{array}{l}\text { Right } \\
\text { lobe }\end{array}$ & Pleomorphic & DOD(3months) & S & \\
\hline & M & 70 & $\begin{array}{l}\text { Right } \\
\text { lobe }\end{array}$ & Pleomorphic & DOD(2.7months) & SUP & \\
\hline & $M$ & 77 & $\begin{array}{l}\text { Right } \\
\text { lobe }\end{array}$ & embryonal & DOD(3months) & SUP & \\
\hline & M & 64 & $\begin{array}{l}\text { Right } \\
\text { lobe }\end{array}$ & NS & NS & S & \\
\hline & M & 70 & Left lobe & NS & NS & S & \\
\hline 1 & $\mathrm{~F}$ & 50 & Left lobe & NS & DOD(2months) & S & 13 \\
\hline 1 & M & 55 & Left lobe & Pleomorphic & DOD(0.75months) & SUP & 14 \\
\hline 1 & M & 51 & Left lobe & Acinar & DOD(12months) & CT & 15 \\
\hline 1 & M & 61 & Left lobe & embryonal & DOD(2months) & CT & 16 \\
\hline 1 & $\mathrm{~F}$ & 66 & $\begin{array}{l}\text { Right } \\
\text { lobe }\end{array}$ & Acinar & NED(9months) & $\mathrm{S}+\mathrm{CT}$ & 17 \\
\hline 1 & $\mathrm{~F}$ & 49 & $\begin{array}{l}\text { Right } \\
\text { lobe }\end{array}$ & embryonal & AWD(4.2months) & CT & 18 \\
\hline 1 & M & 50 & $\begin{array}{l}\text { Right } \\
\text { lobe }\end{array}$ & Acinar & NED(3months) & S & 19 \\
\hline 1 & M & 75 & Left lobe & Acinar & DOD(1months) & CT & $\begin{array}{l}\text { Present } \\
\text { case }\end{array}$ \\
\hline
\end{tabular}

Note. M, male; F, female; NS, not specified; DOD, died of disease; DUD, died of unrelated disease; AWD, alive with disease; NED, no evidence of disease; SUP, supportive; S, surgery; RT, radiotherapy; CT, chemotherapy.

Table 2 Clinicopathological Features and significance of PPRMS in the middle-aged and elderly 


\begin{tabular}{|llll|}
\hline Clinicopathological Features & group & $n$ & $P$ \\
\hline Sex & M & 28 & 0.001 \\
\hline Age & F & 8 & \\
\hline & $45-49$ & 2 & 0.000 \\
\hline Location & $50-69$ & 27 & \\
\hline & $\geq 70$ & 7 & \\
\hline Histology & Left lobe & 21 & 0.317 \\
\hline & Right lobe & 15 & \\
\hline & Pleomorphic & 21 & 0.000 \\
\hline & Acinar & 4 & \\
\hline & embryonal & 4 & \\
\hline & NS & 7 & \\
\hline
\end{tabular}

Note. M, male; F, female; NS, not specified;

\section{Figures}

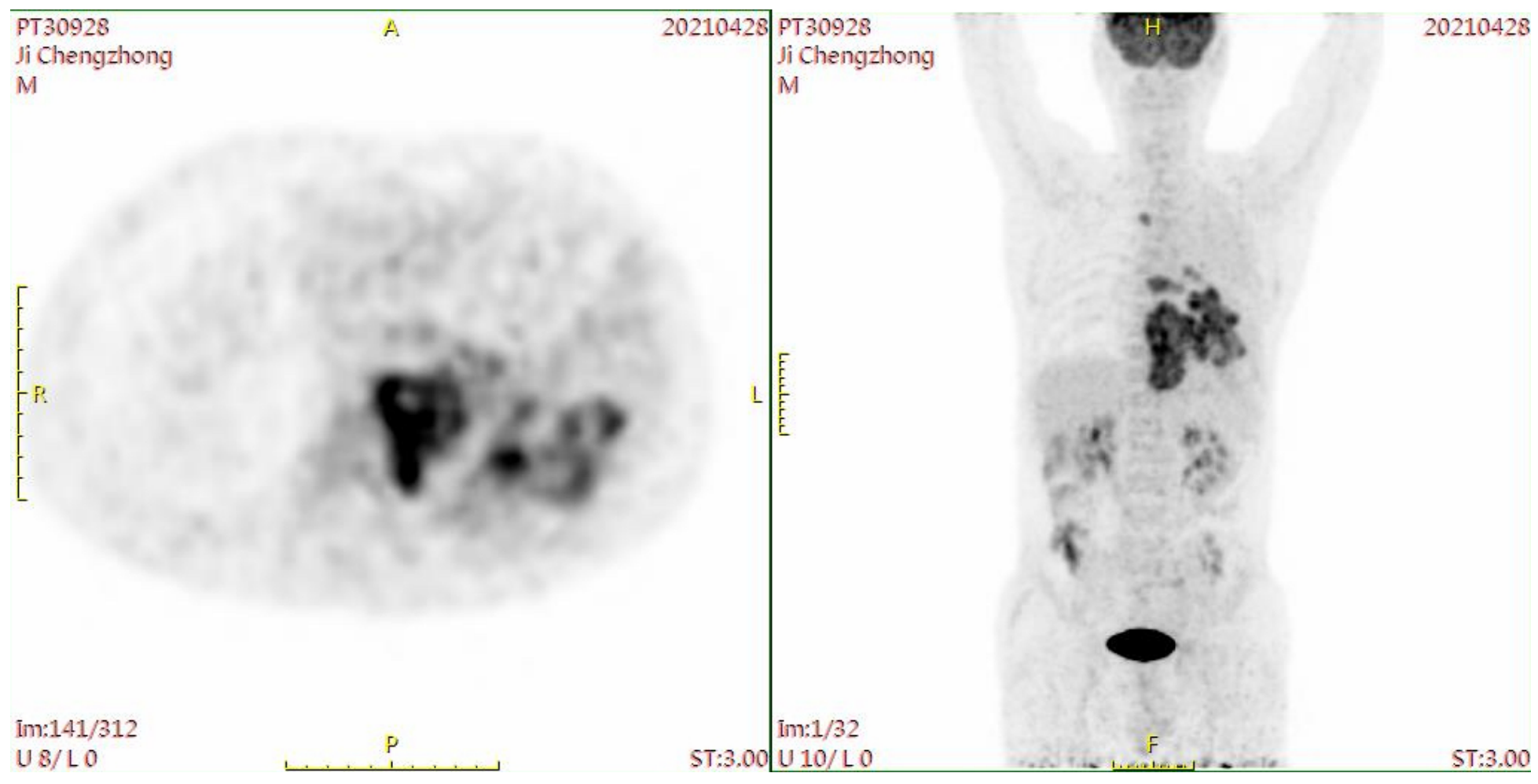

Figure 1 
Transversal (left) and axial (right) section of PET-CT showing a mass in the lung with abnormally high FDG metabolism.
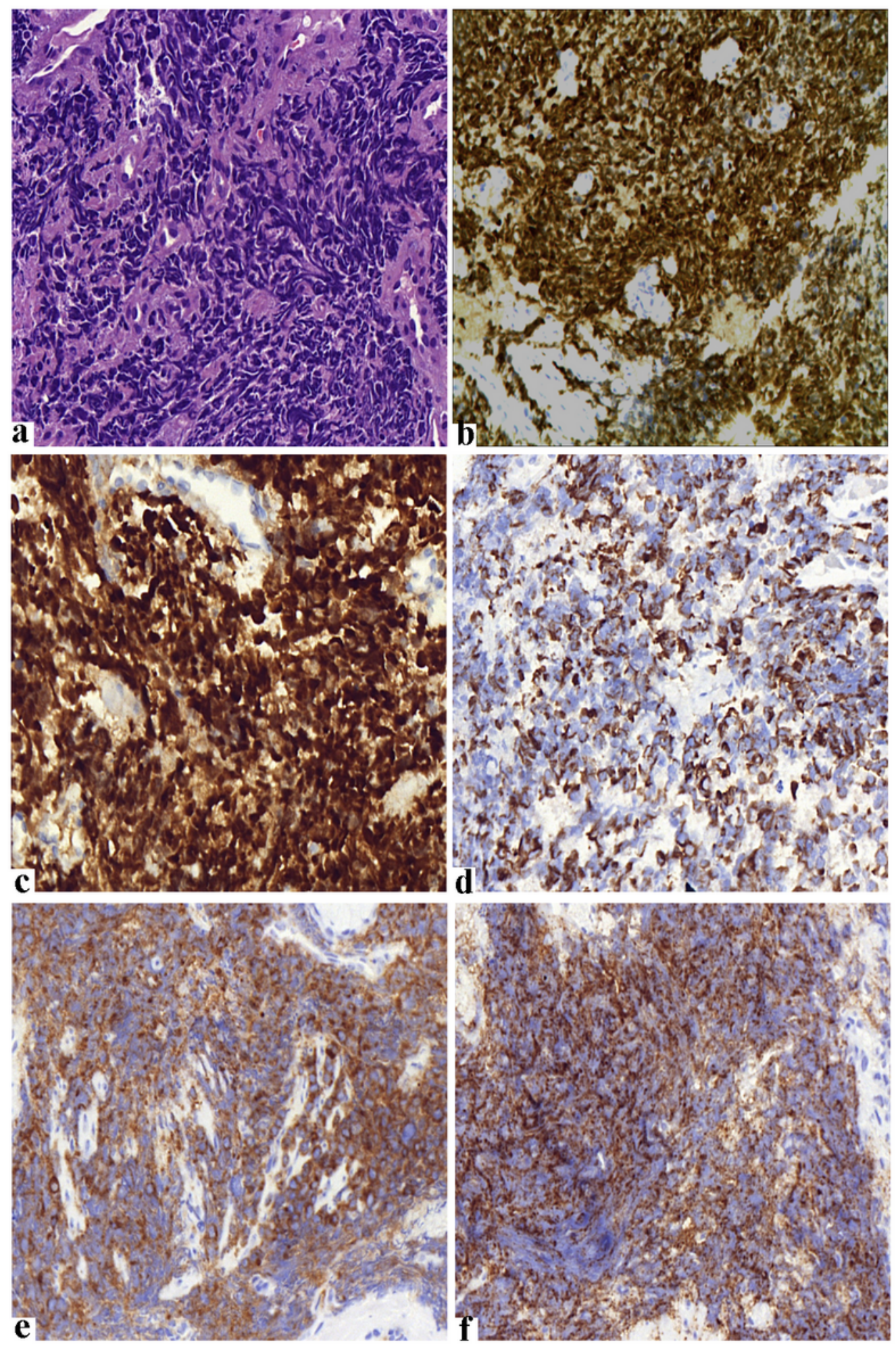

\section{Figure 2}

Acinar RMS showing small tumor cells with few cytoplasm and extrusion deformation caused by mechanical damage resembling SCLC (a H\&E staining, magnification x400). Tumor cells with strong nuclear staining fo MyoD1 (b magnification x400) and Myogenin (c magnification x400) and cytoplasmic 
reactivity for Desmin (d magnification x400), Syn (e magnification x400) and CD56 (f magnification $\mathrm{x} 400)$

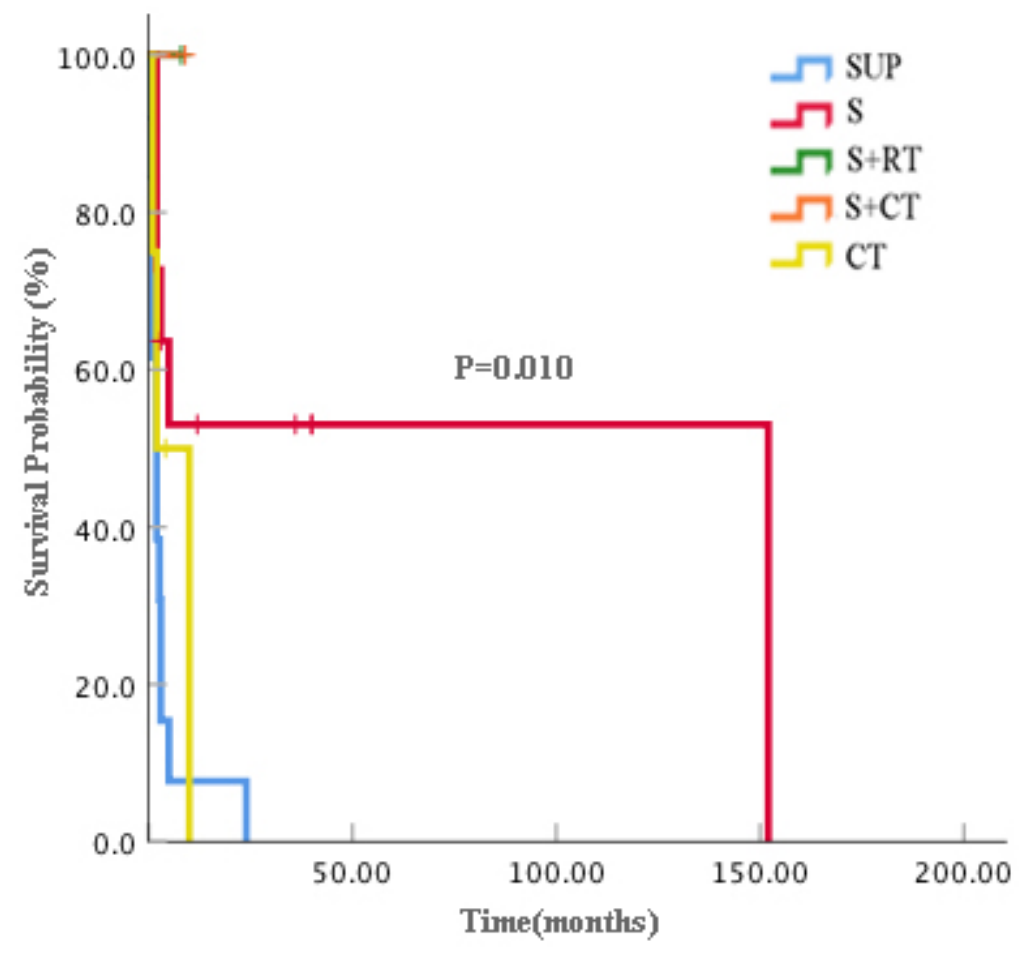

Figure 3

Survival of 35 middle-aged and elderly with RMS diagnosed from 1958-2021 received different treatment methods.

\section{Supplementary Files}

This is a list of supplementary files associated with this preprint. Click to download.

- CAREchecklistEnglish2013.pdf 